\title{
Generalizations of the logarithmic Hardy inequality in critical Sobolev-Lorentz spaces
}

\author{
Shuji Machihara', Tohru Ozawa² and Hidemitsu Wadade ${ }^{3 *}$
}

${ }^{\text {*Correspondence: }}$

wadade@gifu-u.ac.jp

${ }^{3}$ Faculty of Education, Gifu

University, 1-1 Yanagido, Gifu,

501-1193, Japan

Full list of author information is

available at the end of the article

\begin{abstract}
In this paper, we establish the Hardy inequality of the logarithmic type in the critical Sobolev-Lorentz spaces. More precisely, we generalize the Hardy type inequality obtained in Edmunds and Triebel (Math. Nachr. 207:79-92, 1999). The generalized inequality allows us to take the exponents appearing in the inequality more flexibly, and its optimality is discussed in detail. O'Neil's inequality and its reverse play an essential role for the proof.
\end{abstract}

MSC: Primary 46E35; secondary 26D10

Keywords: logarithmic Hardy inequality; critical Sobolev-Lorentz space; O'Neil's inequality

\section{Introduction and the main theorem}

In this paper, we shall give a systematic treatment concerning the Hardy type inequalities on the critical Sobolev-Lorentz spaces $H_{p, q}^{s}\left(\mathbb{R}^{n}\right)$ with $n \in \mathbb{N}, s \in \mathbb{R}, 1<p<\infty$ and $1 \leq q \leq \infty$, where the space $H_{p, q}^{s}\left(\mathbb{R}^{n}\right)$ can be characterized in terms of the Bessel potential such as $H_{p, q}^{s}\left(\mathbb{R}^{n}\right):=(1-\Delta)^{-\frac{s}{2}} L_{p, q}\left(\mathbb{R}^{n}\right)$ with the Lorentz space $L_{p, q}\left(\mathbb{R}^{n}\right)$. We collect precise definitions of those function spaces and related properties in Section 2.

We recall the Sobolev embedding theorem on $H_{p_{1}, p_{2}}^{\frac{n}{p_{1}}}\left(\mathbb{R}^{n}\right)$, which states that the continuous inclusions $H_{p_{1}, p_{2}}^{\frac{n}{p_{1}}}\left(\mathbb{R}^{n}\right) \hookrightarrow L_{q_{1}, q_{2}}\left(\mathbb{R}^{n}\right)$ hold for all $q_{1} \in\left[p_{1}, \infty\right)$ and $q_{2} \in\left[p_{2}, \infty\right]$. However, the limiting case $q_{1}=\infty$ in this embedding fails, provided that $\left(p_{2}, q_{2}\right) \neq(1, \infty)$. This implies that functions in the space $H_{p_{1}, p_{2}}^{\frac{n}{p_{1}}}\left(\mathbb{R}^{n}\right)$ can have a local singularity at some point in $\mathbb{R}^{n}$. In fact, the critical Sobolev space $H_{p}^{\frac{n}{p}}\left(\mathbb{R}^{n}\right)$, which is identical with the critical SobolevLorentz space $H_{p_{1}, p_{2}}^{\frac{n}{p_{1}}}\left(\mathbb{R}^{n}\right)$ with $p_{1}=p_{2}=: p$, admits a singularity of the logarithmic order, see Adams and Fournier [1] and Maz'ya [2]. As a characterization of $H_{p}^{\frac{n}{p}}\left(\mathbb{R}^{n}\right)$, Edmunds and Triebel [3] proved the corresponding Hardy-type inequality with a logarithmic correction as follows.

Theorem A (Edmunds-Triebel [3, Theorem 2.8]) Let $n \in \mathbb{N}$ and $1<p<\infty$. Then there exists a positive constant $C$ such that the inequality

$$
\left(\int_{\left\{|x|<\frac{1}{2}\right\}}\left(\frac{|u(x)|}{|\log | x||}\right)^{p} \frac{d x}{|x|^{n}}\right)^{\frac{1}{p}} \leq C\|u\|_{H_{p}^{\frac{n}{p}}}
$$

holds for all $u \in H_{p}^{\frac{n}{p}}\left(\mathbb{R}^{n}\right)$.

\section{Springer}

(c) 2013 Machihara et al: licensee Springer. This is an Open Access article distributed under the terms of the Creative Commons Attribution License (http://creativecommons.org/licenses/by/2.0), which permits unrestricted use, distribution, and reproduction in any medium, provided the original work is properly cited. 
The main purpose of this paper is to generalize (1.1) into two directions. First, we prove the corresponding logarithmic Hardy-type inequality in the critical Sobolev-Lorentz space $H_{p_{1}, p_{2}}^{\frac{n}{p_{1}}}\left(\mathbb{R}^{n}\right)$, which coincides with (1.1) when $p_{1}=p_{2}=: p$. Furthermore, we investigate the possibility whether the exponents appearing in the inequalities can be taken more flexibly, including the consideration on its optimality. Indeed, our main result now reads as follows.

Theorem 1.1 Let $n \in \mathbb{N}, 1<p<\infty, 1<q \leq \infty$ and $1<\alpha, \beta<\infty$. Then the inequality

$$
\left(\int_{\left\{|x|<\frac{1}{2}\right\}} \frac{|u(x)|^{\alpha}}{|\log | x||^{\beta}} \frac{d x}{|x|^{n}}\right)^{\frac{1}{\alpha}} \leq C\|u\|_{H_{p, q}^{\frac{n}{p}}}
$$

holds for all $u \in H_{p, q}^{\frac{n}{p}}\left(\mathbb{R}^{n}\right)$ if and only if one of the following conditions (i), (ii) and (iii) is fulfilled

$$
\left\{\begin{aligned}
\text { (i) } 1+\alpha-\beta<0 & \\
\text { (ii) } 1+\alpha-\beta \geq 0 \quad \text { and } \quad q<\frac{\alpha}{1+\alpha-\beta} & \text {;ii) } 1+\alpha-\beta>0, \quad q=\frac{\alpha}{1+\alpha-\beta} \text { and } \alpha \geq \beta .
\end{aligned}\right.
$$

Remark 1.2 The condition (ii) in (1.3) allows us to take $1+\alpha-\beta=0$, which implies $\frac{\alpha}{1+\alpha-\beta}=\infty$. In the special case of $p=q=\alpha=\beta$, the inequality (1.2) is precisely inequality (1.1) by Edmunds and Triebel [3]. Also note that the value $q=\frac{\alpha}{1+\alpha-\beta}$ is the critical exponent in the sense that inequality (1.2) holds or not. Moreover, Theorem 1.1 states that when $q=\frac{\alpha}{1+\alpha-\beta}$, inequality (1.2) holds if $\alpha \geq \beta$ and fails if $\alpha<\beta$. In particular, inequality (1.2) fails for the marginal case $q=\infty$ and $1+\alpha-\beta=0$. Indeed, the function $u_{0}$ defined by $u_{0}(x):=\eta(x)|\log | x||$ belongs to $H_{p, \infty}^{\frac{n}{p}}\left(\mathbb{R}^{n}\right)$, where $\eta$ is a cut-off function supported near the origin, while

$$
\int_{\left\{|x|<\frac{1}{2}\right\}} \frac{\left|u_{0}(x)\right|^{\alpha}}{|\log | x||^{1+\alpha}} \frac{d x}{|x|^{n}}=+\infty
$$

There is a number of both mathematical and physical applications of Hardy-type inequalities. Among others, we refer the reader to Adimurthi et al. [4], Beckner [5], Bradley [6], Brézis and Marcus [7], Edmunds and Triebel [3], García and Peral [8], Gurka and Opic [9], Herbst [10], Kalf and Walter [11], Kerman and Pick [12-14], Ladyzhenskaya [15], Machihara et al. [16], Matsumura and Yamagata [17], Nagayasu and Wadade [18], Ozawa and Sasaki [19], Pick [20], Reed and Simon [21], Triebel [22] and Zhang [23]. Especially, in Bradley [6] and Edmunds and Triebel [3], the similar type inequalities to (1.2) were considered in terms of Besov-type spaces.

This paper is organized as follows. Section 2 is devoted to the definition of the SobolevLorentz space, as well as several lemmas needed for the proof of Theorem 1.1. We shall prove Theorem 1.1 in Section 3.

\section{Preliminaries}

In this section, we first recall the definition of the Lorentz spaces. To this end, we define the rearrangement of measurable functions. For a measurable function $f$ on $\mathbb{R}^{n}$ with $n \in \mathbb{N}$, 
$f_{*}:[0, \infty) \rightarrow[0, \infty]$ denotes the distribution function of $f$ given by

$$
f_{*}(\lambda):=\left|\left\{x \in \mathbb{R}^{n} ;|f(x)|>\lambda\right\}\right| \quad \text { for } \lambda \geq 0,
$$

and then the rearrangement $f^{*}:[0, \infty) \rightarrow[0, \infty]$ of $f$ is defined by

$$
f^{*}(t):=\inf \left\{\lambda>0 ; f_{*}(\lambda) \leq t\right\} \quad \text { for } t \geq 0 .
$$

Moreover, $f^{* *}:(0, \infty) \rightarrow[0, \infty]$ denotes the average function of $f^{*}$ defined by

$$
f^{* *}(t):=\frac{1}{t} \int_{0}^{t} f^{*}(\tau) d \tau \quad \text { for } t>0
$$

In what follows, we assume that $f^{*}(t)<+\infty$ for all $t>0$. Then $f^{*}$ is right-continuous and non-increasing on $(0, \infty)$, and hence, $f^{* *}$ is continuous and non-increasing on $(0, \infty)$ with $f^{*}(t) \leq f^{* *}(t)$ for all $t>0$. We now introduce the Lorentz space by using the rearrangement. Let $1 \leq p<\infty$ and $1 \leq q \leq \infty$. Then the Lorentz space $L_{p, q}\left(\mathbb{R}^{n}\right)$ is defined as a function space, equipped with the following norm,

$$
\|f\|_{L_{p, q}}:= \begin{cases}\left(\int_{0}^{\infty}\left(t^{\frac{1}{p}} f^{*}(t)\right)^{q} \frac{d t}{t}\right)^{\frac{1}{q}} & \text { if } 1 \leq q<\infty ; \\ \sup _{t>0}\left(t^{\frac{1}{p}} f^{*}(t)\right) & \text { if } q=\infty .\end{cases}
$$

We can take $f^{*}$ replaced by $f^{* *}$ in definition (2.1) as another equivalent norm on $L_{p, q}\left(\mathbb{R}^{n}\right)$ if $p \neq 1$. Indeed, the following Hardy inequality guarantees its equivalence

$$
\left(\int_{0}^{\infty}\left(\frac{t^{\frac{1}{p}}}{t} \int_{0}^{t} f(\tau) d \tau\right)^{q} \frac{d t}{t}\right)^{\frac{1}{q}} \leq p^{\prime}\left(\int_{0}^{\infty}\left(t^{\frac{1}{p}} f(t)\right)^{q} \frac{d t}{t}\right)^{\frac{1}{q}}
$$

for non-negative measurable functions $f$, for which the integral on the right-hand side in (2.2) is finite. Remark that inequality (2.2) is still valid for the case $q=\infty$ by replacing the integral by the supremum. For the proof of (2.2), see O'Neil [24, Lemma 2.3] and references therein. Furthermore, since $f^{*}$ and $f^{* *}$ are both monotonically non-increasing functions in $(0, \infty)$, we easily get the following decay estimates. For any $t>0$, we have

$$
f^{*}(t) \leq\left(\frac{q}{p}\right)^{\frac{1}{q}} t^{-\frac{1}{p}}\|f\|_{L_{p, q}}
$$

and if $p>1$, together with inequality (2.2), we also have for any $t>0$,

$$
f^{* *}(t) \leq p^{\prime}\left(\frac{q}{p}\right)^{\frac{1}{q}} t^{-\frac{1}{p}}\|f\|_{L_{p, q}}
$$

Note that inequalities (2.3) and (2.4) are also valid for the marginal case $q=\infty$, and we will utilize them frequently for the proof of the main theorem in Section 3.

We also make use of the celebrated Hardy-Littlewood inequality

$$
\int_{\mathbb{R}^{n}}|f(x) g(x)| d x \leq \int_{0}^{\infty} f^{*}(t) g^{*}(t) d t
$$


for all measurable functions $f$ and $g$. The proof of (2.5) can be found in Bennett and Sharpley [25, Theorem 2.2].

Next, we recall the pointwise rearrangement inequality for the convolution of functions proved by O'Neil [24, Theorem 1.7]. In fact, for measurable functions $f$ and $g$ on $\mathbb{R}^{n}$, we have

$$
(f * g)^{* *}(t) \leq t f^{* *}(t) g^{* *}(t)+\int_{t}^{\infty} f^{*}(\tau) g^{*}(\tau) d \tau \quad \text { for } t>0
$$

Moreover, we make use of the reverse O'Neil inequality, established in Kozono et al. [26, Lemma 2.2]. Indeed, there exists a positive constant $C$ such that the inequality

$$
(f * g)^{* *}(t) \geq C\left(t f^{* *}(t) g^{* *}(t)+\int_{t}^{\infty} f^{*}(\tau) g^{*}(\tau) d \tau\right)
$$

holds for all $t>0$ and for all measurable functions $f$ and $g$ on $\mathbb{R}^{n}$, which are both nonnegative, radially symmetric and non-increasing in the radial direction.

In this paper, we frequently use the Bessel potential $G_{s} *:=(1-\Delta)^{-\frac{s}{2}}$ and the Riesz potential $I_{s} *:=(-\Delta)^{-\frac{s}{2}}$ for $0<s<n$. More precisely, the kernel functions $I_{s}$ and $G_{s}$ are defined respectively by

$$
\left\{\begin{array}{l}
I_{s}(x):=\frac{\Gamma\left(\frac{n-s}{2}\right)}{2^{s} \pi^{\frac{n}{2}} \Gamma\left(\frac{s}{2}\right)}|x|^{-(n-s)} \\
G_{s}(x):=\frac{1}{(4 \pi)^{\frac{s}{2}} \Gamma\left(\frac{s}{2}\right)} \int_{0}^{\infty} e^{-\pi \frac{|x|^{2}}{t}-\frac{t}{4 \pi}} t^{-\frac{n-s}{2}} \frac{d t}{t}
\end{array}\right.
$$

for $x \in \mathbb{R}^{n} \backslash\{0\}$, where $\Gamma$ denotes the gamma function. Based on the Lorentz space, we define the Sobolev-Lorentz space $H_{p, q}^{s}\left(\mathbb{R}^{n}\right)$ by $H_{p, q}^{s}\left(\mathbb{R}^{n}\right):=(I-\Delta)^{-\frac{s}{2}} L_{p, q}\left(\mathbb{R}^{n}\right)=G_{s} * L_{p, q}\left(\mathbb{R}^{n}\right)$, equipped with the norm $\|u\|_{H_{p, q}^{s}}:=\left\|(I-\Delta)^{\frac{s}{2}} u\right\|_{L_{p, q}}$. The space $H_{p, q}^{s}\left(\mathbb{R}^{n}\right)$ is a generalization of the usual Sobolev space $H_{p}^{s}\left(\mathbb{R}^{n}\right)$, since we have $L_{p, p}\left(\mathbb{R}^{n}\right)=L_{p}\left(\mathbb{R}^{n}\right)$ due to the norminvariance of $\|u\|_{L_{p, p}}=\|u\|_{L_{p}}$. We now collect the elementary properties of $I_{s}$ and $G_{s}$ in the following lemma.

Lemma 2.1 Let $n \in \mathbb{N}$ and $0<s<n$.

(i) $I_{s}$ and $G_{s}$ are non-negative, radially symmetric and non-increasing in the radial direction, so that $I_{s}^{*}(t)=I_{s}(x)$ and $G_{s}^{*}(t)=G_{s}(x)$ if $|x|=\left(\frac{t}{\omega_{n}}\right)^{\frac{1}{n}}>0$, where $\omega_{n}:=\frac{2 \pi^{\frac{\pi}{2}}}{n \Gamma\left(\frac{n}{2}\right)}$ denotes the volume of the unit ball in $\mathbb{R}^{n}$.

(ii) $G_{s}(x) \leq I_{s}(x)$ for all $x \in \mathbb{R}^{n} \backslash\{0\}$, which implies the $G_{s}^{*}(t) \leq I_{s}^{*}(t), G_{s}^{* *}(t) \leq I_{s}^{* *}(t)$ for all $t>0$, and $\lim _{|x| \downarrow 0} \frac{G_{s}(x)}{I_{s}(x)}=\lim _{t \downarrow 0} \frac{G_{s}^{*}(t)}{I_{s}^{*}(t)}=1$.

(iii) $\left\|G_{s}\right\|_{L_{1}}=1$ and there exists a positive constant $C$ such that the following inequalities hold

$$
G_{s}(x) \leq \begin{cases}C|x|^{-(n-s)} & \text { for } x \in \mathbb{R}^{n} \backslash\{0\} \\ C e^{-|x|} & \text { for } x \in \mathbb{R}^{n} \text { with }|x| \geq 1\end{cases}
$$

Since the facts in Lemma 2.1 are well known, we omit the detailed proof here, see Stein [27], for instance. Furthermore, we refer to Almgren and Lieb [28] and Bennett and Sharpley [25] for further information about the rearrangement theory. 
In the end of this section, we shall show the following one-dimensional Hardy inequality of logarithmic type.

Lemma 2.2 Let $1<\alpha, \beta<\infty$. Then there exists a positive constant $C$ such that the inequality

$$
\left(\int_{0}^{\frac{1}{2}}\left(\int_{t}^{\frac{1}{2}}|\phi(s)| d s\right)^{\alpha}|\log t|^{-\beta} \frac{d t}{t}\right)^{\frac{1}{\alpha}} \leq C\left(\int_{0}^{\frac{1}{2}}|\phi(t)|^{\alpha}|\log t|^{-\beta} \frac{d t}{t}\right)^{\frac{1}{\alpha}}
$$

holds for all measurable functions $\phi$ such that the integral on the right-hand side of (2.8) is finite.

Furthermore, we can show the following dual variant of inequality (2.8).

Lemma 2.3 Let $1<\beta \leq \alpha<\infty$ and $q:=\frac{\alpha}{1+\alpha-\beta}$. Then there exists a positive constant $C$ such that the inequality

$$
\left(\int_{0}^{\frac{1}{2}}\left(\int_{t}^{\frac{1}{2}}|\phi(s)| d s\right)^{\alpha}|\log t|^{-\beta} \frac{d t}{t}\right)^{\frac{1}{\alpha}} \leq C\left(\int_{0}^{\frac{1}{2}}(t|\phi(t)|)^{q} \frac{d t}{t}\right)^{\frac{1}{q}}
$$

holds for all measurable functions $\phi$ such that the integral on the right-hand side of (2.9) is finite.

We shall apply Lemma 2.3 for the proof of the sufficiency part of Theorem 1.1 in Section 3, and Lemma 2.2 will be used for the proof of the necessity part of Theorem 1.1 in Section 4. Lemma 2.2 and Lemma 2.3 can be obtained as corollaries of the following weighted inequalities obtained in Bradley [6] and Muckenhoupt [29].

Theorem B (Bradley [6], Muckenhoupt [29]) Let $1<\rho \leq \sigma<\infty$ and let $U$ and $V$ be measurable weights.

(i) There exists a positive constant $C$ such that the inequality

$$
\left(\int_{0}^{\infty}\left|U(t) \int_{0}^{t}\right| \psi(s)|d s|^{\sigma} d t\right)^{\frac{1}{\sigma}} \leq C\left(\int_{0}^{\infty}|V(t) \psi(t)|^{\rho} d t\right)^{\frac{1}{\rho}}
$$

holds for all measurable functions $\psi$ such that the integral on the right-hand side of (2.10) is finite if and only if

$$
\sup _{r>0}\left(\int_{r}^{\infty}|U(t)|^{\sigma} d t\right)^{\frac{1}{\sigma}}\left(\int_{0}^{r}|V(t)|^{-\rho^{\prime}} d t\right)^{\frac{1}{\rho^{\prime}}}<+\infty .
$$

(ii) There exists a positive constant $C$ such that the inequality

$$
\left(\int_{0}^{\infty}\left|U(t) \int_{t}^{\infty}\right| \psi(s)|d s|^{\sigma} d t\right)^{\frac{1}{\sigma}} \leq C\left(\int_{0}^{\infty}|V(t) \psi(t)|^{\rho} d t\right)^{\frac{1}{\rho}}
$$


holds for all measurable functions $\psi$ such that the integral on the right-hand side of (2.11) is finite if and only if

$$
\sup _{r>0}\left(\int_{0}^{r}|U(t)|^{\sigma} d t\right)^{\frac{1}{\sigma}}\left(\int_{r}^{\infty}|V(t)|^{-\rho^{\prime}} d t\right)^{\frac{1}{\rho^{\prime}}}<+\infty .
$$

Now we shall show Lemma 2.2 and Lemma 2.3 by applying Theorem B(i) and Theorem $B(i i)$, respectively.

Proof of Lemma 2.2 Define the weights $U_{1}$ and $V_{1}$ by

$$
U_{1}(t):= \begin{cases}|\log t|^{-\frac{\beta}{\alpha}} t^{-\frac{1+\alpha}{\alpha}} & \text { for } 0<t<\frac{1}{2} \\ 0 & \text { for } t \geq \frac{1}{2}\end{cases}
$$

and

$$
V_{1}(t):= \begin{cases}|\log t|^{-\frac{\beta}{\alpha}} t^{-\frac{1}{\alpha}} & \text { for } 0<t<\frac{1}{2} \\ 1 & \text { for } t \geq \frac{1}{2} .\end{cases}
$$

Then the direct calculation shows

$$
\sup _{r>0}\left(\int_{r}^{\infty}\left|U_{1}(t)\right|^{\alpha} d t\right)^{\frac{1}{\alpha}}\left(\int_{0}^{r}\left|V_{1}(t)\right|^{-\alpha^{\prime}} d t\right)^{\frac{1}{\alpha^{\prime}}}<+\infty
$$

Thus, Theorem B(i) implies that

$$
\left(\int_{0}^{\infty}\left|U_{1}(t) \int_{0}^{t}\right| \psi(s)|d s|^{\alpha} d t\right)^{\frac{1}{\alpha}} \leq C\left(\int_{0}^{\infty}\left|V_{1}(t) \psi(t)\right|^{\alpha} d t\right)^{\frac{1}{\alpha}}
$$

namely,

$$
\begin{aligned}
& \left(\int_{0}^{\frac{1}{2}}\left(\frac{1}{t} \int_{0}^{t}|\psi(s)| d s\right)^{\alpha}|\log t|^{-\beta} \frac{d t}{t}\right)^{\frac{1}{\alpha}} \\
& \quad \leq C\left(\int_{0}^{\frac{1}{2}}|\psi(t)|^{\alpha}|\log t|^{-\beta} \frac{d t}{t}+\int_{\frac{1}{2}}^{\infty}|\psi(t)|^{\alpha} d t\right)^{\frac{1}{\alpha}}
\end{aligned}
$$

for all measurable functions $\psi$. Taking $\phi=\chi_{\left(0, \frac{1}{2}\right)} \psi$ yields the desired inequality (2.8).

Proof of Lemma 2.3 Define the weights $U_{2}$ and $V_{2}$ by

$$
U_{2}(t):= \begin{cases}|\log t|^{-\frac{\beta}{\alpha}} t^{-\frac{1}{\alpha}} & \text { for } 0<t<\frac{1}{2} \\ 0 & \text { for } t \geq \frac{1}{2}\end{cases}
$$

and

$$
V_{2}(t):= \begin{cases}t^{\frac{q-1}{q}} & \text { for } 0<t<\frac{1}{2} \\ t^{\frac{2 q-1}{q}} & \text { for } t \geq \frac{1}{2}\end{cases}
$$


Then the direct calculation shows

$$
\sup _{r>0}\left(\int_{0}^{r}\left|U_{2}(t)\right|^{\alpha} d t\right)^{\frac{1}{\alpha}}\left(\int_{r}^{\infty}\left|V_{2}(t)\right|^{-q^{\prime}} d t\right)^{\frac{1}{q^{\prime}}}<+\infty
$$

Since $\alpha \geq \beta$ implies $\alpha \geq q$, by applying Theorem $\mathrm{B}(\mathrm{ii})$, we obtain

$$
\begin{aligned}
& \left(\int_{0}^{\frac{1}{2}}\left(\int_{t}^{\infty}|\psi(s)| d s\right)^{\alpha}|\log t|^{-\beta} \frac{d t}{t}\right)^{\frac{1}{\alpha}} \\
& \quad \leq C\left(\int_{0}^{\frac{1}{2}}(t|\psi(t)|)^{q} \frac{d t}{t}+\int_{\frac{1}{2}}^{\infty} t^{2 q-1}|\psi(t)|^{q} d t\right)^{\frac{1}{q}}
\end{aligned}
$$

for all measurable functions $\psi$. Taking $\phi=\chi_{\left(0, \frac{1}{2}\right)} \psi$ yields the desired inequality (2.9).

\section{Proof of the sufficiency part of Theorem 1.1}

In this section, we consider the sufficiency part of Theorem 1.1. To this end, it suffices to show the following key lemmas.

Lemma 3.1 Let $n \in \mathbb{N}, 1<p<\infty, 1<q \leq \infty$, and let $1<\alpha, \beta<\infty$. Assume one of the conditions (i), (ii) and (iii) in (1.3). Then there exists a positive constant $C$ such that the inequality

$$
\left(\int_{0}^{\frac{1}{2}} \frac{u^{*}(t)^{\alpha}}{|\log t|^{\beta}} \frac{d t}{t}\right)^{\frac{1}{\alpha}} \leq C\|u\|_{H_{p, q}^{\frac{n}{p}}}
$$

holds for all $u \in H_{p, q}^{\frac{n}{p}}\left(\mathbb{R}^{n}\right)$.

Lemma 3.2 Let $n \in \mathbb{N}, 1<p<\infty, 1<q \leq \infty$, and let $1<\alpha, \beta<\infty$. Assume one of the conditions (i), (ii) and (iii) in (1.3). Then there exists a positive constant $C$ such that the inequality

$$
\left(\int_{\mathbb{R}^{n}}|w(x) u(x)|^{\alpha} d x\right)^{\frac{1}{\alpha}} \leq C\left(\sup _{0<t<\frac{1}{2}} t^{\frac{1}{\alpha}}|\log t|^{\frac{\beta}{\alpha}} w^{*}(t)\right)\|u\|_{H_{p, q}^{\frac{n}{p}}}
$$

holds for all $u \in H_{p, q}^{\frac{n}{p}}\left(\mathbb{R}^{n}\right)$ and for all measurable function $w$ satisfying

$$
|\operatorname{supp} w|<\frac{1}{2} \quad \text { and } \quad \sup _{0<t<\frac{1}{2}} t^{\frac{1}{\alpha}}|\log t|^{\frac{\beta}{\alpha}} w^{*}(t)<\infty .
$$

Remark 3.3 By taking $w(x):=|\log | x||^{-\frac{\beta}{\alpha}}|x|^{-\frac{n}{\alpha}} \chi_{\{|x|<\varepsilon\}}(x)$ with small $\varepsilon>0$ in Lemma 3.2, we can prove the sufficiency part of Theorem 1.1, where $\chi_{\{|x|<\varepsilon\}}$ is a characteristic function on $\{|x|<\varepsilon\}$.

First, we shall prove Lemma 3.2 by applying Lemma 3.1 . 
Proof of Lemma 3.2 By using inequality (2.5) with $|\operatorname{supp} w|<\frac{1}{2}$ and applying Lemma 3.1, we see

$$
\begin{aligned}
\int_{\mathbb{R}^{n}}|w(x) u(x)|^{\alpha} d x & =\int_{0}^{\frac{1}{2}}(w u)^{*}(t)^{\alpha} d t \leq \int_{0}^{\frac{1}{2}} w^{*}(t)^{\alpha} u^{*}(t)^{\alpha} d t \\
& =\int_{0}^{\frac{1}{2}}\left(t^{\frac{1}{\alpha}}|\log t|^{\frac{\beta}{\alpha}} w^{*}(t)\right)^{\alpha} \frac{u^{*}(t)^{\alpha}}{|\log t|^{\beta}} \frac{d t}{t} \\
& \left.\leq \sup _{0<t<\frac{1}{2}} t|\log t|^{\beta} w^{*}(t)^{\alpha}\right) \int_{0}^{\frac{1}{2}} \frac{u^{*}(t)^{\alpha}}{|\log t|^{\beta}} \frac{d t}{t} \\
& \leq C\left(\sup _{0<t<\frac{1}{2}} t|\log t|^{\beta} w^{*}(t)^{\alpha}\right)\|u\|_{H_{p, q}^{\frac{n}{p}}}^{\alpha}
\end{aligned}
$$

which is exactly the inequality (3.2).

We are now in a position to prove Lemma 3.1.

Proof of Lemma 3.1 First, by letting $(1-\Delta)^{\frac{n}{2 p}} u=f \in L_{p, q}\left(\mathbb{R}^{n}\right)$, Lemma 3.1 can be rewritten as the following equivalent form

$$
\left(\int_{0}^{\frac{1}{2}} \frac{\left(G_{\frac{n}{p}} * f\right)^{*}(t)^{\alpha}}{|\log t|^{\beta}} \frac{d t}{t}\right)^{\frac{1}{\alpha}} \leq C\|f\|_{L_{p, q}}
$$

for $f \in L_{p, q}\left(\mathbb{R}^{n}\right)$. Hence, we concentrate our attention on the proof of (3.3) below. By the O'Neil inequality (2.6) and decay estimates (2.3) and (2.4), we have for $0<t<\frac{1}{2}$,

$$
\begin{aligned}
& \left(G_{\frac{n}{p}} * f\right)^{*}(t) \\
& \quad \leq\left(G_{\frac{n}{p}} * f\right)^{* *}(t) \\
& \quad \leq t G_{\frac{n}{p}}^{* *}(t) f^{* *}(t)+\int_{t}^{\infty} G_{\frac{n}{p}}^{*}(s) f^{*}(s) d s \\
& \quad t G_{\frac{n}{p}}^{* *}(t) f^{* *}(t)+\int_{\frac{1}{2}}^{\infty} G_{\frac{n}{p}}^{*}(s) f^{*}(s) d s+\int_{t}^{\frac{1}{2}} G_{\frac{n}{p}}^{*}(s) f^{*}(s) d s \\
& \quad \leq C\left(\left\|G_{\frac{n}{p}}\right\|_{L_{p^{\prime}, \infty}}\|f\|_{L_{p, q}}+\left\|G_{\frac{n}{p}}\right\|_{L_{1}}\|f\|_{L_{p, q}} \int_{\frac{1}{2}}^{\infty} s^{-\left(1+\frac{1}{p}\right)} d s\right)+\int_{t}^{\frac{1}{2}} G_{\frac{n}{p}}^{*}(s) f^{*}(s) d s \\
& \quad=C\|f\|_{L_{p, q}}+\int_{t}^{\frac{1}{2}} G_{\frac{n}{p}}^{*}(s) f^{*}(s) d s .
\end{aligned}
$$

Thus, from (3.4), we obtain

$$
\begin{aligned}
\left(\int_{0}^{\frac{1}{2}} \frac{\left(G_{\frac{n}{p}} * f\right)^{*}(t)^{\alpha}}{|\log t|^{\beta}} \frac{d t}{t}\right)^{\frac{1}{\alpha}} \leq & C\left(\int_{0}^{\frac{1}{2}}|\log t|^{-\beta} \frac{d t}{t}\right)^{\frac{1}{\alpha}}\|f\|_{L_{p, q}} \\
& +\left(\int_{0}^{\frac{1}{2}}\left(\int_{t}^{\frac{1}{2}} G_{\frac{n}{p}}^{*}(s) f^{*}(s) d s\right)^{\alpha}|\log t|^{-\beta} \frac{d t}{t}\right)^{\frac{1}{\alpha}},
\end{aligned}
$$

where the integral of the first term on the right-hand side of (3.5) is finite since $\beta>1$. We further estimate the integral of the second term below. 
Note that the conditions (i), (ii) and (iii) in (1.3) can be rewritten equivalently as follows

(i) $\beta>1+\frac{\alpha}{q^{\prime}}$ or (ii) $1+\frac{\alpha}{q^{\prime}}=\beta$ and $\alpha \geq \beta$.

Case 1. Assume (i) in (3.6). For $0<t<\frac{1}{2}$, by Lemma 2.1(ii) and Hölder's inequality, we see

$$
\begin{aligned}
\int_{t}^{\frac{1}{2}} G_{\frac{n}{p}}^{*}(s) f^{*}(s) d s & \leq C \int_{t}^{\frac{1}{2}} s^{\frac{1}{p}} f^{*}(s) \frac{d s}{s} \\
& \leq C\left(\int_{t}^{\frac{1}{2}} \frac{d s}{s}\right)^{\frac{1}{q^{\prime}}}\left(\int_{t}^{\frac{1}{2}}\left(s^{\frac{1}{p}} f^{*}(s)\right)^{q} \frac{d s}{s}\right)^{\frac{1}{q}} \leq C|\log t|^{\frac{1}{q^{\prime}}}\|f\|_{L_{p, q}} .
\end{aligned}
$$

Note that the calculation above is also valid for the case $q=\infty$. Thus, we have

$$
\begin{aligned}
& \left(\int_{0}^{\frac{1}{2}}\left(\int_{t}^{\frac{1}{2}} G_{\frac{n}{p}}^{*}(s) f^{*}(s) d s\right)^{\alpha}|\log t|^{-\beta} \frac{d t}{t}\right)^{\frac{1}{\alpha}} \\
& \quad \leq C\left(\int_{0}^{\frac{1}{2}}|\log t|^{\frac{\alpha}{q^{\prime}}-\beta} \frac{d t}{t}\right)^{\frac{1}{\alpha}}\|f\|_{L_{p, q}} \leq C\|f\|_{L_{p, q}},
\end{aligned}
$$

where we have used the condition $\beta>1+\frac{\alpha}{q}$, which ensures that the integral on the middlehand side of (3.7) is finite. Thus, combining (3.5) with (3.7), we obtain the desired estimate.

Case 2. Assume (ii) in (3.6). By Lemma 2.1(ii) and Lemma 2.3, we have

$$
\begin{aligned}
& \left(\int_{0}^{\frac{1}{2}}\left(\int_{t}^{\frac{1}{2}} G_{\frac{n}{p}}^{*}(s) f^{*}(s) d s\right)^{\alpha}|\log t|^{-\beta} \frac{d t}{t}\right)^{\frac{1}{\alpha}} \\
& \quad \leq C\left(\int_{0}^{\frac{1}{2}}\left(t G_{\frac{n}{p}}^{*}(t) f^{*}(t)\right)^{q} \frac{d t}{t}\right)^{\frac{1}{q}} \\
& \quad \leq C\left(\int_{0}^{\frac{1}{2}}\left(t^{\frac{1}{p}} f^{*}(t)\right)^{q} \frac{d t}{t}\right)^{\frac{1}{q}} \leq C\|f\|_{L_{p, q} .}
\end{aligned}
$$

Thus, combining (3.5) with (3.8), we obtain the desired estimate.

\section{Proof of the necessity part of Theorem 1.1}

In this final section, we shall prove the necessity part of Theorem 1.1. To this end, we shall construct a concrete function in the critical Sobolev-Lorentz space $H_{p, q}^{\frac{n}{p}}\left(\mathbb{R}^{n}\right)$.

Proof of the necessity part of Theorem 1.1 First, by putting $(1-\Delta)^{\frac{n}{2 p}} u=f$, inequality (1.2) can be rewritten as

$$
\left(\int_{\left\{|x|<\frac{1}{2}\right\}} \frac{\left|G_{\frac{n}{p}} * f(x)\right|^{\alpha}}{|\log | x||^{\beta}} \frac{d x}{|x|^{n}}\right)^{\frac{1}{\alpha}} \leq C\|f\|_{L_{p, q}} .
$$


Therefore, it is enough to show the breakdown of the inequality (4.1) under the following conditions, which are the negations of (1.3) or (3.6),

$$
\left\{\begin{aligned}
\text { (i) } & \beta<1+\frac{\alpha}{q^{\prime}} \text { and } q<\infty ; \\
\text { (ii) } & \beta \leq 1+\frac{\alpha}{q^{\prime}}(=1+\alpha) \text { and } q=\infty ; \\
\text { (iii) } & \beta=1+\frac{\alpha}{q^{\prime}}, \quad q<\infty \text { and } \alpha<\beta .
\end{aligned}\right.
$$

Case 1. Assume (i) in (4.2). In this case, we define the function $f_{\varepsilon}$ by

$$
f_{\varepsilon}(x):=|\log | x||^{-\frac{1+\varepsilon}{q}}|x|^{-\frac{n}{p}} \chi_{\{|x|<\varepsilon\}}(x)
$$

for small $\varepsilon>0$. Then we see that for sufficiently small $\varepsilon>0, f_{\varepsilon}$ becomes non-negative and non-increasing with respect to the radial direction $|x|$. Thus, we have for small $t>0$

$$
f_{\varepsilon}^{*}(t)=\tilde{f}_{\varepsilon}\left(\left(\frac{t}{\omega_{n}}\right)^{\frac{1}{n}}\right) \simeq|\log t|^{-\frac{1+\varepsilon}{q}} t^{-\frac{1}{p}}=: g_{\varepsilon}(t)
$$

where $\tilde{f}_{\varepsilon}(|x|):=f_{\varepsilon}(x)$. More precisely, (4.4) implies that there exist positive constants $\delta$ small enough, $C$ and $\tilde{C}$ such that the inequalities

$$
C g_{\varepsilon}(t) \leq f_{\varepsilon}^{*}(t) \leq \tilde{C} g_{\varepsilon}(t)
$$

hold for all $0<t<\delta$. By using (4.5), it is easy to see $f_{\varepsilon} \in L_{p, q}\left(\mathbb{R}^{n}\right)$. Indeed, from (4.5), we obtain

$$
\int_{0}^{\delta}\left(t^{\frac{1}{p}} f_{\varepsilon}^{*}(t)\right)^{q} \frac{d t}{t} \leq \tilde{C} \int_{0}^{\delta}\left(t^{\frac{1}{p}} g_{\varepsilon}(t)\right)^{q} \frac{d t}{t}=\tilde{C} \int_{0}^{\delta}|\log t|^{-(1+\varepsilon)} \frac{d t}{t}<\infty
$$

On the other hand, since $f_{\varepsilon}$ is non-negative and non-increasing with respect to the radial direction, so is $G_{\frac{n}{p}} * f_{\varepsilon}$. Thus, noting $G_{\frac{n}{p}} * f_{\varepsilon}(x)=\left(G_{\frac{n}{p}} * f_{\varepsilon}\right)^{*}\left(\omega_{n} r^{n}\right)$ if $|x|=r>0$, we see by changing a variable $\omega_{n} r^{n}=t$,

$$
\begin{aligned}
& \int_{\left\{|x|<\frac{1}{2}\right\}} \frac{\left|G_{\frac{n}{p}} * f_{\varepsilon}(x)\right|^{\alpha}}{|\log | x||^{\beta}} \frac{d x}{|x|^{n}} \\
& =n \omega_{n} \int_{0}^{\frac{1}{2}} \frac{\left(G_{\frac{n}{p}} * f_{\varepsilon}\right)^{*}\left(\omega_{n} r^{n}\right)^{\alpha}}{|\log r|^{\beta}} \frac{d r}{r} \\
& \geq C \int_{0}^{\delta} \frac{\left(G_{\frac{n}{p}} * f_{\varepsilon}\right)^{*}(t)^{\alpha}}{|\log t|^{\beta}} \frac{d t}{t}
\end{aligned}
$$

for small $\delta>0$. Furthermore, by using Lemma 2.2 and the reverse O'Neil inequality (2.7), we have

$$
\begin{aligned}
& \int_{0}^{\delta} \frac{\left(G_{\frac{n}{p}} * f_{\varepsilon}\right)^{*}(t)^{\alpha}}{|\log t|^{\beta}} \frac{d t}{t} \\
& \quad \geq C \int_{0}^{\delta} \frac{\left(G_{\frac{n}{p}} * f_{\varepsilon}\right)^{* *}(t)^{\alpha}}{|\log t|^{\beta}} \frac{d t}{t}
\end{aligned}
$$




$$
\begin{aligned}
& \geq C \int_{0}^{\delta} \frac{\left(t G_{\frac{n}{p}}^{* *}(t) f_{\varepsilon}^{* *}(t)+\int_{t}^{\infty} G_{\frac{n}{p}}^{*}(\tau) f_{\varepsilon}^{*}(\tau) d \tau\right)^{\alpha}}{|\log t|^{\beta}} \frac{d t}{t} \\
& \geq C \int_{0}^{\delta} \frac{\left(\int_{t}^{\delta} G_{\frac{n}{p}}^{*}(\tau) f_{\varepsilon}^{*}(\tau) d \tau\right)^{\alpha}}{|\log t|^{\beta}} \frac{d t}{t} .
\end{aligned}
$$

Thus, by Lemma 2.1 (ii) and (4.5), we have for small $\delta>0$,

$$
\begin{aligned}
\int_{0}^{\delta} \frac{\left(\int_{t}^{\delta} G_{\frac{n}{p}}^{*}(\tau) f_{\varepsilon}^{*}(\tau) d \tau\right)^{\alpha}}{|\log t|^{\beta}} \frac{d t}{t} & \geq C \int_{0}^{\delta} \frac{\left(\int_{t}^{\delta} I_{\frac{n}{p}}^{*}(\tau) f_{\varepsilon}^{*}(\tau) d \tau\right)^{\alpha}}{|\log t|^{\beta}} \frac{d t}{t} \\
& \geq C \int_{0}^{\delta} \frac{\left(\int_{t}^{\delta} \tau^{-\frac{1}{p^{\prime}}} g_{\varepsilon}(\tau) d \tau\right)^{\alpha}}{|\log t|^{\beta}} \frac{d t}{t}
\end{aligned}
$$

Take $\varepsilon>0$ small enough, so that $1-\frac{1+\varepsilon}{q}>0$, which is possible since $q>1$. Thus, we have for any $0<t<\frac{\delta}{2}$ with small $\delta>0$,

$$
\int_{t}^{\delta} \tau^{-\frac{1}{p^{\prime}}} g_{\varepsilon}(\tau) d \tau=\frac{q}{q-(1+\varepsilon)}\left(|\log t|^{1-\frac{1+\varepsilon}{q}}-|\log \delta|^{1-\frac{1+\varepsilon}{q}}\right) \geq C|\log t|^{1-\frac{1+\varepsilon}{q}} .
$$

Summing up all estimates (4.6), (4.7), (4.8), and (4.9), we obtain

$$
\int_{\left\{|x|<\frac{1}{2}\right\}} \frac{\left|G_{\frac{n}{p}} * f_{\varepsilon}(x)\right|^{\alpha}}{|\log | x||^{\beta}} \frac{d x}{|x|^{n}} \geq C \int_{0}^{\frac{\delta}{2}}|\log t|^{\left(1-\frac{1+\varepsilon}{q}\right) \alpha-\beta} \frac{d t}{t} .
$$

However, the integral on the right-hand side of (4.10) diverges, provided that $\varepsilon>0$ is taken small enough, so that $\left(1-\frac{1+\varepsilon}{q}\right) \alpha-\beta+1 \geq 0$, which is possible since $\frac{\alpha}{q^{\prime}}-\beta+1>0$ by the assumption. Thus, inequality (4.1) fails under the condition (i) in (4.2).

Case 2. Assume (ii) in (4.2). In this case, we utilize $f_{0}(x):=|x|^{-\frac{n}{p}}$ instead of $f_{\varepsilon}(x)$ used in Case 1 . Then it is easily seen $f_{0} \in L_{p, \infty}\left(\mathbb{R}^{n}\right)$. On the other hand, in a quite similar way carried out in Case 1, we see

$$
\begin{aligned}
\int_{\left\{|x|<\frac{1}{2}\right\}} \frac{\left|G_{\frac{n}{p}} * f_{0}(x)\right|^{\alpha}}{|\log | x||^{\beta}} \frac{d x}{|x|^{n}} & \geq C \int_{0}^{\delta} \frac{\left(G_{\frac{n}{p}} * f_{0}\right)^{*}(t)^{\alpha}}{|\log t|^{\beta}} \frac{d t}{t} \\
& \geq C \int_{0}^{\delta} \frac{\left(G_{\frac{n}{p}} * f_{0}\right)^{* *}(t)^{\alpha}}{|\log t|^{\beta}} \frac{d t}{t} \geq C \int_{0}^{\delta} \frac{\left(\int_{t}^{\delta} G_{\frac{n}{p}}^{*}(\tau) f_{0}^{*}(\tau) d \tau\right)^{\alpha}}{|\log t|^{\beta}} \frac{d t}{t} \\
& \geq C \int_{0}^{\delta} \frac{\left(\int_{t}^{\delta} I_{\frac{n}{p}}^{*}(\tau) f_{0}^{*}(\tau) d \tau\right)^{\alpha}}{|\log t|^{\beta}} \frac{d t}{t} \geq C \int_{0}^{\frac{\delta}{2}}|\log t|^{\alpha-\beta} \frac{d t}{t}
\end{aligned}
$$

for small $\delta$, where the last integral diverges if $\alpha-\beta+1 \geq 0$, that is, $\beta \leq 1+\alpha$. Thus, inequality (4.1) fails under the condition (ii) in (4.2).

Case 3. Assume (iii) in (4.2), which implies that $\frac{\alpha}{q}=1+\alpha-\beta<1$, namely, $q>\alpha$. In this case, we make use of the function $f_{\varepsilon}$ with small $\varepsilon>0$ defined by

$$
f_{\varepsilon}(x):=|\log | x||^{-\frac{1}{q}}|\log | \log |x|||^{-\frac{1+\varepsilon}{q}}|x|^{-\frac{n}{p}} \chi_{\{|x|<\varepsilon\}}(x)
$$


Since $f_{\varepsilon}$ is non-negative and non-increasing in the radial direction $|x|$ with small $\varepsilon>0$, we see

$$
f_{\varepsilon}^{*}(t) \simeq|\log t|^{-\frac{1}{q}}|\log | \log t||^{-\frac{1+\varepsilon}{q}} t^{-\frac{1}{p}}=: g_{\varepsilon}(t)
$$

for small $t>0$, namely, there exist positive constants $\delta$ small enough, $C$ and $\tilde{C}$ such that the inequalities

$$
C g_{\varepsilon}(t) \leq f_{\varepsilon}^{*}(t) \leq \tilde{C} g_{\varepsilon}(t)
$$

hold for all $0<t<\delta$. By using (4.11), it is easy to see $f_{\varepsilon} \in L_{p, q}\left(\mathbb{R}^{n}\right)$. Indeed,

$$
\begin{aligned}
\int_{0}^{\delta}\left(t^{\frac{1}{p}} f_{\varepsilon}^{*}(t)\right)^{q} \frac{d t}{t} & \leq \tilde{C} \int_{0}^{\delta}\left(t^{\frac{1}{p}} g_{\varepsilon}(t)\right)^{q} \frac{d t}{t} \\
& =\tilde{C} \int_{0}^{\delta}|\log t|^{-1}|\log | \log t||^{-(1+\varepsilon)} \frac{d t}{t}<\infty
\end{aligned}
$$

On the other hand, in the same estimates from below as in (4.6), (4.7) and (4.8) in Case 1, we obtain

$$
\int_{\left\{|x|<\frac{1}{2}\right\}} \frac{\left|G_{\bar{p}} * f_{\varepsilon}(x)\right|^{\alpha}}{|\log | x||^{\beta}} \frac{d x}{|x|^{n}} \geq C \int_{0}^{\delta} \frac{\left(\int_{t}^{\delta} \tau^{-\frac{1}{p^{\prime}}} g_{\varepsilon}(\tau) d \tau\right)^{\alpha}}{|\log t|^{\beta}} \frac{d t}{t}
$$

Furthermore, we can easily see

$$
\int_{t}^{\delta} \tau^{-\frac{1}{p^{\prime}}} g_{\varepsilon}(\tau) d \tau=\left.\int_{t}^{\delta}|\log \tau|^{-\frac{1}{q}}|\log | \log \tau||^{-\frac{1+\varepsilon}{q}} \frac{d \tau}{\tau} \simeq|\log t|^{1-\frac{1}{q}}|\log | \log t\right|^{-\frac{1+\varepsilon}{q}}
$$

for small $t>0$. In particular, for any $0<t<\frac{\delta}{2}$ with small $\delta>0$, we have

$$
\int_{t}^{\delta} \tau^{-\frac{1}{p^{\prime}}} g_{\varepsilon}(\tau) d \tau \geq C|\log t|^{1-\frac{1}{q}}|\log | \log t||^{-\frac{1+\varepsilon}{q}}
$$

Thus, combining (4.12) with (4.13), we see

$$
\begin{aligned}
\int_{\left\{|x|<\frac{1}{2}\right\}} \frac{\left|G_{\frac{n}{p}} * f_{\varepsilon}(x)\right|^{\alpha}}{|\log | x||^{\beta}} \frac{d x}{|x|^{n}} & \geq C \int_{0}^{\frac{\delta}{2}} \frac{\left(\int_{t}^{\delta} \tau^{-\frac{1}{p^{\prime}}} g_{\varepsilon}(\tau) d \tau\right)^{\alpha}}{|\log t|^{\beta}} \frac{d t}{t} \\
& \geq\left. C \int_{0}^{\frac{\delta}{2}}|\log t|^{\frac{\alpha}{q^{\prime}}-\beta}|\log | \log t\right|^{-\frac{1+\varepsilon}{q} \alpha} \frac{d t}{t} \\
& =\left.C \int_{0}^{\frac{\delta}{2}}|\log t|^{-1}|\log | \log t\right|^{-\frac{1+\varepsilon}{q} \alpha} \frac{d t}{t}
\end{aligned}
$$

However, the last integral in (4.14) diverges, provided that $\varepsilon>0$ is taken small, so that $-\frac{1+\varepsilon}{q} \alpha+1 \geq 0$, which is possible since $q>\alpha$. Thus, the inequality (4.1) fails under the condition (iii) in (4.2). 
Remark 4.1 (3.1) in Lemma 3.1 is equivalent to (1.2) in Theorem 1.1. Indeed, we have already seen in Section 3 that Lemma 3.1 implies Theorem 1.1. On the other hand, (1.2) is equivalent to (4.1), and since the weighted norm in the left-hand side of (4.1) is nondecreasing under the rearrangement, (4.1) can be reduced to (3.3), which is equivalent to (3.1).

\section{Competing interests}

The authors declare that they have no competing interests.

\section{Authors' contributions}

HW drafted the manuscript. All authors computed to complete the proof of main theorems, and they read and approved the final manuscript.

\section{Author details}

${ }^{1}$ Faculty of Education, Saitama University, 255 Shimookubo, Saitama, Sakuraku 338-8570, Japan. ${ }^{2}$ Department of Applied Physics, Waseda University, Shinjuku, Tokyo 169-8555, Japan. ${ }^{3}$ Faculty of Education, Gifu University, 1-1 Yanagido, Gifu, 501-1193, Japan

\section{Acknowledgements}

The authors are grateful to the referees for their valuable comments.

Received: 6 February 2013 Accepted: 31 July 2013 Published: 14 August 2013

\section{References}

1. Adams, RA, Fournier, JJF: Sobolev Spaces, 2nd edn. Academic Press, Amsterdam (2003)

2. Maz'ya, VG: Sobolev Spaces. Springer, Berlin (1985)

3. Edmunds, DE, Triebel, H: Sharp Sobolev embeddings and related Hardy inequalities: the critical case. Math. Nachr. 207, 79-92 (1999)

4. Adimurthi, Chaudhuri, N, Ramaswamy, M: An improved Hardy-Sobolev inequality and its application. Proc. Am. Math. Soc. 130, 489-505 (2002)

5. Beckner, W: Pitt's inequality with sharp convolution estimates. Proc. Am. Math. Soc. 136, 1871-1885 (2008)

6. Bradley, JS: Hardy inequalities with mixed norms. Can. Math. Bull. 21, 405-408 (1978)

7. Brézis, H, Marcus, M: Hardy's inequalities revisited. Ann. Sc. Norm. Super. Pisa, Cl. Sci. 25, 217-237 (1997)

8. García, JPA, Peral, IA: Hardy inequalities and some critical elliptic and parabolic problems. J. Differ. Equ. 144, 441-476 (1998)

9. Gurka, P, Opic, B: Sharp embeddings of Besov-type spaces. J. Comput. Appl. Math. 208, 235-269 (2007)

10. Herbst, WI: Spectral theory of the operator $\left(p^{2}+m^{2}\right)^{1 / 2}-Z e^{2} / r$. Commun. Math. Phys. 53, 285-294 (1977)

11. Kalf, $\mathrm{H}$, Walter, J: Strongly singular potentials and essential self-adjointness of singular elliptic operators in $C_{0}^{\infty}\left(\mathbb{R}^{n} \backslash\{0\}\right)$. J. Funct. Anal. 10, 114-130 (1972)

12. Kerman, R, Pick, L: Optimal Sobolev embeddings. Forum Math. 18, 535-570 (2006)

13. Kerman, R, Pick, L: Optimal Sobolev embedding spaces. Stud. Math. 192, 195-217 (2009)

14. Kerman, R, Pick, L: Explicit formulas for optimal rearrangement-invariant norms in Sobolev imbedding inequalities. Stud. Math. 206, 97-119 (2011)

15. Ladyzhenskaya, OA: The Mathematical Theory of Viscous Incompressible Flow. Second English edition, revised and enlarged. Translated from the Russian by Richard A. Silverman and John Chu. Mathematics and its Applications, vol. 2. Gordon \& Breach, New York (1969)

16. Machihara, S, Ozawa, T, Wadade, H: Hardy type inequalities on balls. Tohoku Math. J. (to appear)

17. Matsumura, A, Yamagata, N: Global weak solutions of the Navier-Stokes equations for multidimensional compressible flow subject to large external potential forces. Osaka J. Math. 38, 399-418 (2001)

18. Nagayasu, S, Wadade, $\mathrm{H}$ : Characterization of the critical Sobolev space on the optimal singularity at the origin. J. Funct. Anal. 258, 3725-3757 (2010)

19. Ozawa, T, Sasaki, H: Inequalities associated with dilations. Commun. Contemp. Math. 11, 265-277 (2009)

20. Pick, L: Optimal Sobolev embeddings. In: Nonlinear Analysis, Function Spaces and Applications (Prague, 1998), vol. 6, pp. 156-199. Acad. Sci. Czech Repub., Prague (1999)

21. Reed, M, Simon, B: Methods of Modern Mathematical Physics. Il. Fourier Analysis, Self-Adjointness. Academic Press, New York (1975)

22. Triebel, H: Sharp Sobolev embeddings and related Hardy inequalities: the sub-critical case. Math. Nachr. 208, 167-178 (1999)

23. Zhang, J: Extensions of Hardy inequality. J. Inequal. Appl. 2006, Article ID 69379 (2006)

24. O'Neil, R: Convolution operators and $L(p, q)$ spaces. Duke Math. J. 30, 129-142 (1963)

25. Bennett, C, Sharpley, R: Interpolation of Operators. Academic Press, New York (1988)

26. Kozono, H, Sato, T, Wadade, H: Upper bound of the best constant of a Trudinger-Moser inequality and its application to a Gagliardo-Nirenberg inequality. Indiana Univ. Math. J. 55, 1951-1974 (2006)

27. Stein, EM: Singular Integrals and Differentiability Properties of Functions. Princeton University Press, Princeton (1970)

28. Almgren, FJ, Lieb, EH: Symmetric decreasing rearrangement is sometimes continuous. J. Am. Math. Soc. 2, 683-773 (1989)

29. Muckenhoupt, B: Hardy's inequality with weights. Stud. Math. 44, 31-38 (1972) 
doi:10.1186/1029-242X-2013-381

Cite this article as: Machihara et al.: Generalizations of the logarithmic Hardy inequality in critical Sobolev-Lorentz spaces. Journal of Inequalities and Applications 2013 2013:381.

Submit your manuscript to a SpringerOpen ${ }^{\circ}$ journal and benefit from:

- Convenient online submission

- Rigorous peer review

- Immediate publication on acceptance

Open access: articles freely available online

- High visibility within the field

- Retaining the copyright to your article

Submit your next manuscript at $\gg$ springeropen.com 